\title{
The Response of Suprachiasmatic Neurons of the Rat Hypothalamus to Photic and Nicotinic Stimuli
}

\author{
J. D. Miller, D. M. Murakami, and C. A. Fuller \\ Department of Animal Physiology, University of California, Davis, California 95616
}

\begin{abstract}
Single neurons in the suprachiasmatic nucleus (SCN) and surrounding hypothalamic nuclei of the rat were characterized functionally and pharmacologically. Photically responsive neurons of the SCN typically exhibited a similar response to intravenous nicotine. The response to nicotine was antagonized by the nicotinic blocker, mecamylamine. Nicotinic blockade either eliminated or even reversed the sign of the photic response during subsequent photic stimulation. This constellation of results was not seen in neurons of adjacent hypothalamic regions.
\end{abstract}

The suprachiasmatic nucleus ( $\mathrm{SCN}$ ) of the hypothalamus is a critical locus for the maintenance and entrainment of circadian rhythms. The essential role of the SCN in circadian timekeeping is indicated by the observation that SCN lesions result in the disruption of the majority of endogenous behavioral and hormonal rhythms (e.g., Stephan and Zucker, 1972; Moore and Eichler, 1976). Such endogenous rhythms are most effectively entrained by light (Moore-Ede et al., 1982). A sufficient pathway for the 7eitgeber effects of visual stimulation appears to be the retinohypothalamic projection to the $\mathrm{SCN}$, since interruption of other retinal efferents does not prevent photic entrainment (Rusak, 1977).

Certain other stimuli can mimic the entraining effects of light on circadian rhythms. For instance, direct electrical stimulation of the $\mathrm{SCN}$ can generate large phase shifts in the circadian rhythms of feeding and locomotor behavior (Rusak and Groos, 1982). Similarly, intraventricular administration of a cholinergic agonist causes substantial phase shifts in circadian rhythms (Zatz and Herkenham, 1981; Earnest and Turek, 1985). Furthermore, the inhibitory effect of cholinergic agonists on the nocturnal elevation of pineal $5-\mathrm{H} T \mathrm{~N}$-acetyltransferase is blocked by the simultaneous application of D-tubocurarine, a nicotinic antagonist, but not atropinc, a muscarinic antagonist. This result suggests that the cholinergic effects are highly specific and are mediated by a nicotinic rather than muscarinic receptor.

Photic and cholinergic stimuli apparently interact at some point along the retinohypothalamic pathway. Photic stimulation causes a 3-fold increase in ACh in the SCN, and the normal circadian rhythm of ACh in the SCN is abolished by ocular enucleation (Murakami et al., 1984). The application of the nicotine antagonist, $\alpha$-bungarotoxin, to the SCN blocks the inhibitory effects of light on the nocturnal elevation of pineal 5-HT

\footnotetext{
Received Mar. 24, 1986; revised Sept. 2, 1986; accepted Sept. 8, 1986.

This work was supported by NIH Grant MH41477. We wish to thank Melissa Crawford and Andrea Lauber for technical support and Linda Cotner for preparation of the manuscript.

Correspondence should be addressed to Charles A. Fuller at the above address. Copyright (C) 1987 Society for Neuroscience $0270-6474 / 87 / 040978-09 \$ 02.00 / 0$
}

$\mathrm{N}$-acetyltransferase (Zatz and Brownstein, 1981). Finally, intraventricular administration of mecamylamine, another nicotinic antagonist, blocks the entraining effects of light pulses on the wheel-running circadian rhythm in hamsters (F. Turek, personal communication, 1985). Such results suggest that the relationship between photic and cholinergic effects on circadian rhythms may be mediated by a nicotinic receptor at some level of the retinohypothalamic pathway.

At a more molecular level many single neurons of the SCN are responsive to the effects of retinal illumination (Nishino et al., 1976; Groos and Mason, 1978, 1980; Kreisel et al., 1979; Sawaki, 1979; Inouye and Kawamura, 1982). Nishino et al. (1976) found twice as many SCN neurons that were excited by visual stimulation or electrical stimulation of the optic nerve than were inhibited by such stimulation in the male rat. Groos and Mason $(1978,1980)$ confirmed this finding and also showed that the predominant response (excitatory or inhibitory) was rather sluggish and tonic, as opposed to phasic. Such cells exhibited rather large receptive fields, with no obvious centersurround organization.

Relatively little is known of the pharmacological characteristics of SCN neurons. The SCN is heavily labeled by the putative nicotinic ligand, $\alpha$-bungarotoxin (Segal et al., 1978; Block and Billiar, 1981). An unambiguous demonstration of a cholinergic projection to the SCN has recently been obtained using a sensitive antibody against the cholinergic marker enzyme, CAT (Ichikawa and Hirata, 1986). In addition, more than $80 \%$ of SCN neurons recorded in vivo are excited by the iontophoretic administration of ACh (Nishino and Koizumi, 1977). Interestingly, the same authors showed that $65 \%$ of SCN neurons responsive to electrical stimulation of the optic tract are also responsive to cholinergic stimulation. Thus, electrophysiological and pharmacological data suggest a close association between photic and cholincrgic ncuronal responses in the SCN, perhaps analogous to that observed at more molar levels (see above). Furthermore, the behavioral and autoradiographic data suggest that the cholinergic response may be mediated by a nicotinic receptor. This study examined possible neuronal mechanisms mediating the photic-cholinergic interaction within the circadian timekeeping system. To characterize further the response profile of SCN neurons and neurons in the surrounding hypothalamus, this study examined the relationship between the photic response of such neurons and their response to intravenous nicotine. Finally, the neuronal photic response in the presence of the nicotinic antagonist, mecamylamine, was examined.

\section{Materials and Methods}

Male Wistar and Long-Evans rats (200-300 gm) were used in this study. Animals were maintained on a 12:12 light/dark cycle at all times. In- 

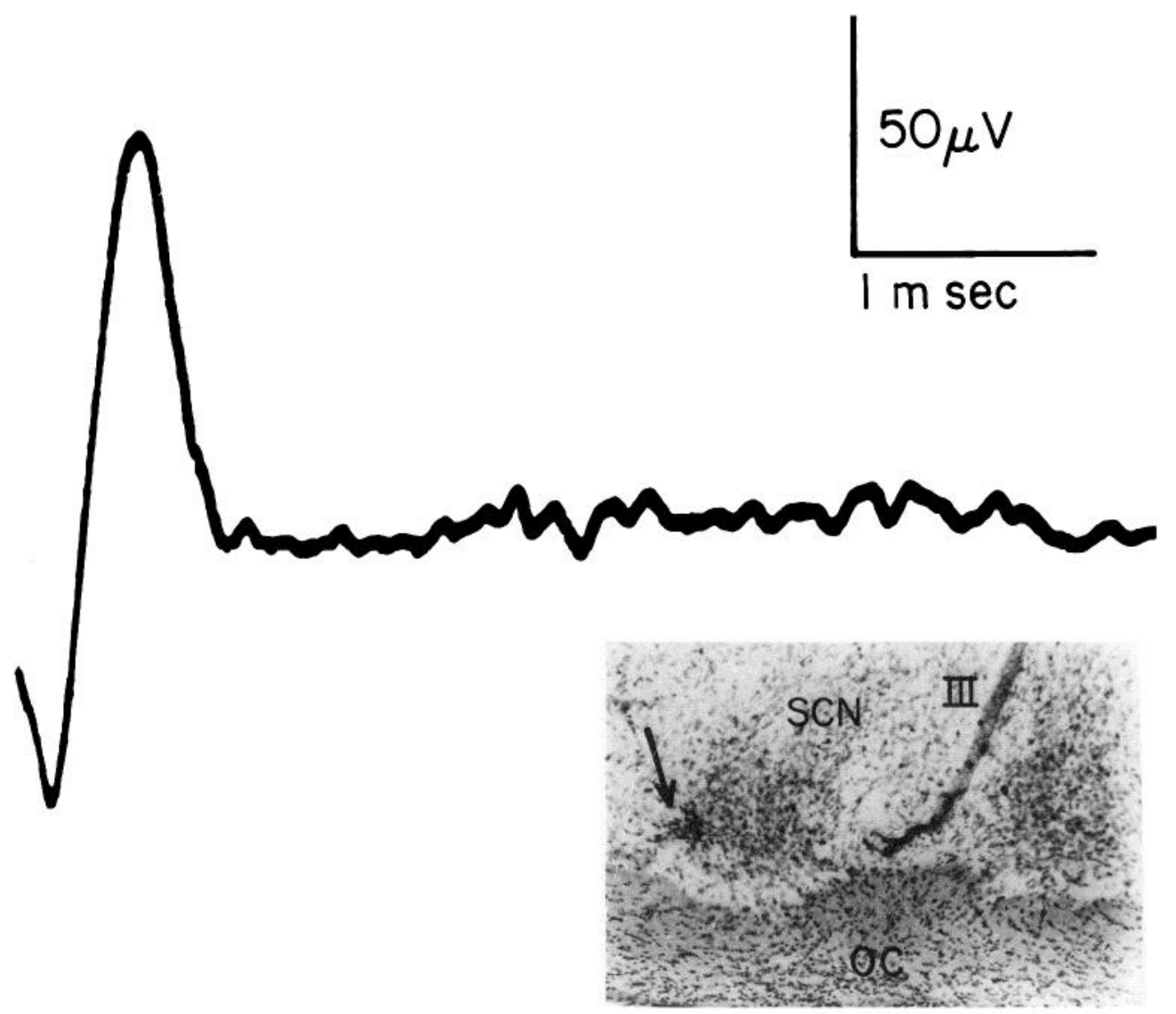

Figure 1. Single action potential recorded from rat SCN. Insert, Typical recording site in the ventrolateral SCN. Arrow points to fast green dye spot. $O C$, Optic chiasm; $I I I$, third ventricle.

dividual rats were anesthetized at about $2 \mathrm{hr}$ after lights-on with urethane $(1.6 \mathrm{gm} / \mathrm{kg})$ after pretreatment with the peripheral muscarinic antagonist, atropine methyl nitrate $(0.5 \mathrm{mg} / \mathrm{kg})$, to reduce respiratory difficulty. The femoral vein was then catheterized for intravenous drug administration. The animals were stereotaxically prepared, utilizing the skull coordinate system of Paxinos and Watson (1982). A skull hole was drilled over the midline at an anterior-posterior coordinate corresponding to $85 \%$ of the distance between ear bar zero and bregma. The sagittal sinus was tied off anteriorly and posteriorly and then sectioned in order to expose the longitudinal sulcus.

Electrode tracks were run through the SCN and surrounding areas at approximately $7.7 \mathrm{~mm}$ anterior, $0.1 \mathrm{~mm}$ lateral to the midline, and 0.5 $1 \mathrm{~mm}$ dorsal to interaural zero. Tungsten microelectrodes (3-10 M $)$ or glass microelectrodes (3-10 M $\Omega$ ) were employed. Electrophysiological signals were passed through a preamplifer, displayed on-line on a storage oscilloscope, and permanently recorded on magnetic tape for off-line analysis. Action potentials were monitored with a rate meter and an audio monitor, and passed through a window discriminator to a minicomputer for rate analysis. Pupils were dilated with topically applied atropine methyl nitrate $(0.5 \mathrm{mg} / \mathrm{ml})$ and coated with a protective silicone solution. Bilateral or ipsilateral (to the electrode placement) retinal illumination ( 85 footcandles) was provided by a fiberoptics system.

Cells in the SCN region were sampled until a photically responsive cell was encountered. The consistency of the photic response was determined with a second presentation of the visual stimulus. Duration of the visual stimulus was typically $2 \mathrm{~min}$. Baseline recording periods and interstimulus intervals were typically $20 \mathrm{~min}$ in duration. Once a photically responsive cell was characterized, the response to $20 \mu \mathrm{g} / \mathrm{kg}$ nicotine administered intravenously was examined. After at least $5 \mathrm{~min}$ the nicotinic antagonist mecamylamine $(0.5-1 \mathrm{mg} / \mathrm{kg})$ was administered. In some experiments further doses of nicotine $(20 \mu \mathrm{g} / \mathrm{kg})$ were administered to examine the effectiveness of the nicotinic blockade. Finally, the neuronal response to photic stimulation following nicotinic blockade was examined. Either a microlesion $(+10 \mu \mathrm{A}$ for $2 \mathrm{~min})$ or a dye spot $(-19 \mu \mathrm{A}$ for $10 \mathrm{~min})$ was then made at the recording site.

The rat was perfused with normal saline, followed by $10 \%$ formalin. The brain was removed, postfixed in $10 \%$ formalin, sectioned at $40 \mu \mathrm{m}$, and stained for Nissl substance with thionin in order to determine electrode locus.

The effect of the experimental manipulations on individual cells was determined by $t$ test. Each $t$ test evaluated the effect of each experimental treatment on the cell's firing rate during the manipulation as compared to an immediately preceding baseline period of equal duration. Each $t$ score represented change in neuronal response indexed to the intrinsic variability in firing rate (baseline), thus avoiding the typically high variance of raw neuronal scores or the extreme sensitivity to baseline changes evidenced by percentage change from baseline scores. Each $t$ score was then standardized (Winer, 1962) to correct for differing degrees of freedom in order to allow comparison of different cells. The averages of the absolute values of $t$ scores for single cells within the SCN proper, and in a group of SCN "misses" (NONSCN) beyond the Nissl defined borders of the $\mathrm{SCN}$ were calculated for each experimental treatment. Absolute value scores gave an index of cellular responsivity not affected by the sign (excitatory or inhibitory) of the response to photic or nicotinic stimulation.

All other analyses were performed on the signed standardized $t$ 's for each cell. Discriminant analysis was performed in order to verify that the anatomically defined cellular groups could be functionally differentiated on the basis of the available dependent variables and to classify a set of "cells" that were difficult to classify as SCN hits or misses on the basis of histological examination. Pearson simple correlation was performed on the standardized $t$ scores to determine the degree of covariation of photic and nicotinic response in the $\mathrm{SCN}$ and non-SCN cell populations. Two-way analysis of variance examined the effects of nicotinic blockade on photic response for SCN and non-SCN cells.

\section{Results}

\section{Baseline characteristics}

Single-cell action potentials recorded from the SCN and periSCN regions were at least $50 \mu \mathrm{v}$ in amplitude (signal-to-noise ratio, $\simeq 2 / 1$ ) with durations of approximately $2 \mathrm{msec}$. A typical single action potential is shown in Figure 1, with the recording 

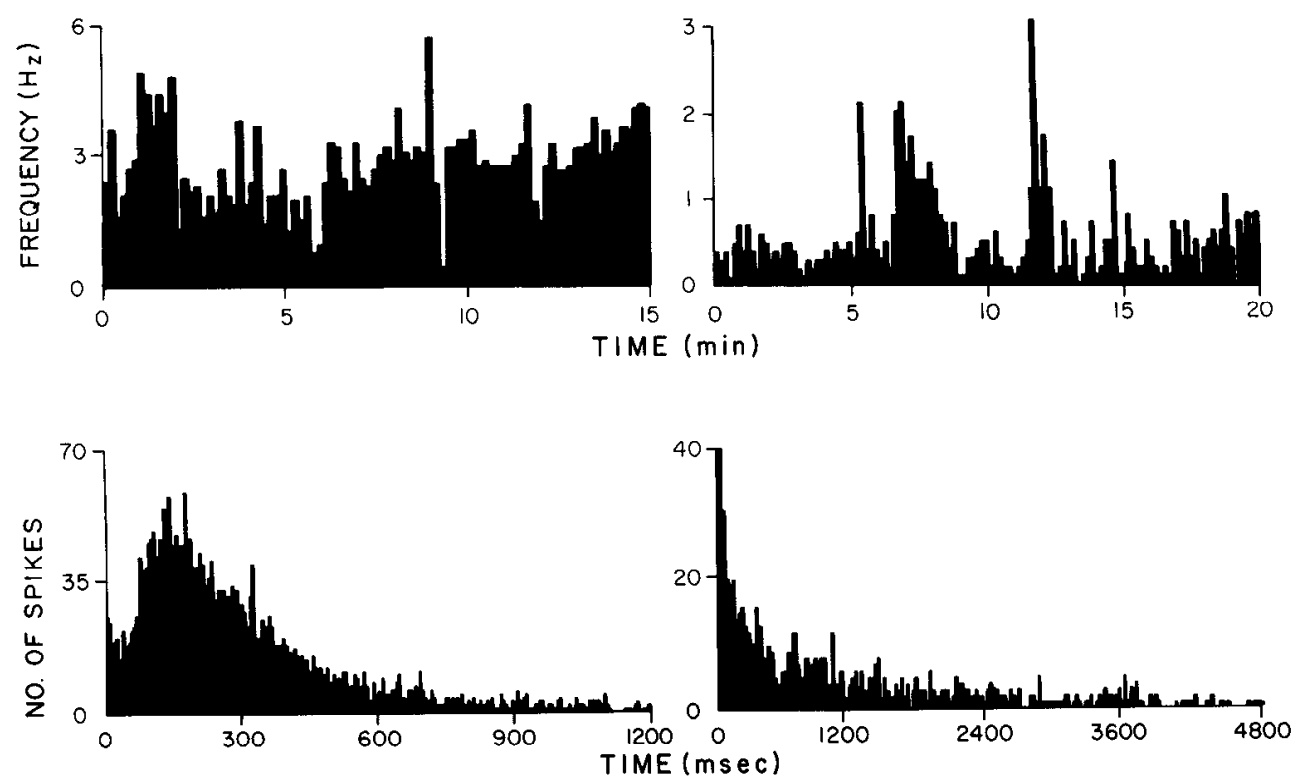

Figure 2. Baseline firing rate histograms (top) and interspike interval histograms (bottom) for 2 individual SCN cells $(A, B)$. In the rate histogram the number of action potentials $/ 10 \mathrm{sec}$ interval is plotted versus time (in $\mathrm{min}$ ). The baseline interspike interval histogram plots the number of action potentials per $5 \mathrm{msec}$ bins (left) and $25 \mathrm{msec}$ bins (right) versus time (in $\mathrm{msec}$ ).

site shown in the inset. Baseline firing rates varied between 0.1 and $11 \mathrm{~Hz}$, with a grand mean of $2.5 \pm 0.4 \mathrm{~Hz}$. The mean firing rate of cells in the SCN $(N=25)$ proper did not differ significantly from that of cells $(N=23)$ in the peri-SCN region (SCN $\bar{X}=2.8 \pm 0.5 \mathrm{~Hz}$, NONSCN $\bar{X}=2.2 \pm 0.5 \mathrm{~Hz}$ ). Typical rate (top) and interspike interval (bottom) histograms for 2 SCN neurons are shown in Figure 2, $A$ and $B$. It is apparent from this figure that baseline firing rates in the $\mathrm{SCN}$ are low and quite variable. The distribution of interspike intervals for SCN neurons is typically skewed to the right, indicating that the firing pattern of such neurons is characterized by frequent "pauses" of varying duration between spikes.

In addition, a small group $(N=4)$ of action potentials were recorded with short durations ( $<1 \mathrm{msec}$ ) and relatively high and steady firing rates $(\bar{X}=5.3 \mathrm{~Hz})$ from histological sites adjacent to or within the optic chiasm. Such potentials probably represented the activity of chiasmatic fibers.

\section{Photic response}

The absolute level of photic response for cells belonging to the SCN group, irrespective of sign (excitatory or inhibitory) was quite high $(t=4.0, p<0.001)$. The absolute level of photic response in the NONSCN group was considerably lower but still significantly different from baseline $(t=2.5, p=0.02)$. An excitatory response to photic stimulation was about 3 times as likely as an inhibitory response ( $n=5$ for inhibitors, $n=14$ for excitors, $n=6$ for no clear response) in the SCN group. Excitatory responses in the NONSCN group were about twice as frequent as inhibitory responses, with many cells exhibiting no response whatsoever to light ( $n=6$ for excitors, $n=3$ for inhibitors, $n=14$ with no clear response). Typical responses to photic stimulation are shown in Figure 3. Although spike amplitude was not consistently quantified in this study, individual cases of amplitude reduction in response to visual stimulation were observed.

\section{Nicotinic response}

The absolute level of nicotinic response in both groups was quite high $(t=4.8, p<0.001$ for SCN; $t=4.6, p<0.001$ for
NONSCN). The predominant response to nicotine in both groups was excitatory ( $n=14$ excitatory, $n=3$ inhibitory for SCN; $n-12$ excitatory, $n=2$ inhibitory for NONSCN). Figure $4 B$ illustrates a typical excitatory response to nicotine. As with photic stimulation, individual cells occasionally exhibited decrements in spike amplitude following administration of intravenous nicotine (Fig. $4 B$ ).

A relatively high correlation $(r=0.42, N=25, p<0.02)$ between photic response and nicotinic response was observed in the SCN group. Although several cells $(N=4)$ in the antcrior hypothalamus and medial preoptic region were similarly responsive to both photic and nicotinic stimulation, the overall correlation between photic and nicotinic response in the NONSCN group was not significant $(r=0.13, N=24)$. Scatter plots of the relationship between photic and nicotinic response for SCN and NONSCN cells are shown in Figure 5. In this figure, the regression line for the NONSCN cells is nearly flat, with the majority of them clustering around zero on the $X$ axis (i.e., cells varying in response to nicotine often exhibited little response to visual stimulation). In contrast, the regression line for the SCN cells has a substantial positive slope and the relationship between photic and nicotinic response is roughly linear, as evidenced by the significant correlation coefficient (see above).

\section{Response to mecamylamine}

The absolute level of response to mecamylamine was highly significant in the SCN group $(t=4.8, p<0.001)$. In the NONSCN group, the response was somewhat smaller but still significant $(t=3.5, p<0.01)$. Mecamylamine had a normalizing effect on cell firing. If nicotine excited the cell, mecamylamine reduced its firing and vice versa. In nearly all cases, the post-mecamylamine firing rate was very close to pre-nicotine baseline $[t=$ 0.9 , not significant (n.s.) for SCN; $t=0.07$, n.s. for NONSCN) In addition to its normalizing effect on firing rate, mecamylamine also exhibited the ability to reverse nicotine-induced alterations in spike amplitude (not shown). Mecamylamine effectively blocked the response of SCN neurons to further doses of nicotine in all cases observed. 


\section{BASELINE}

A

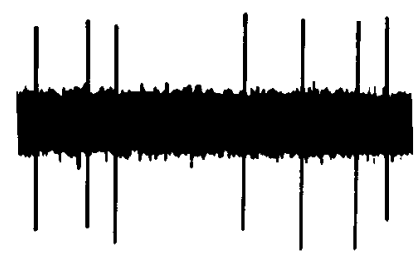

B

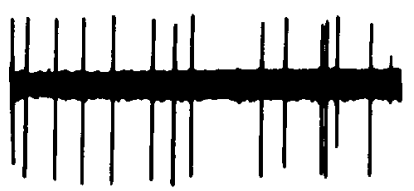

C

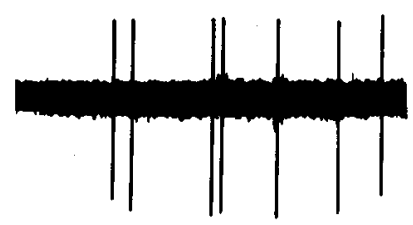

D

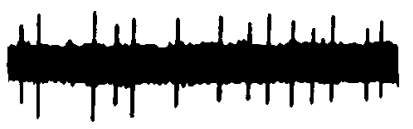

LIGHTS-ON
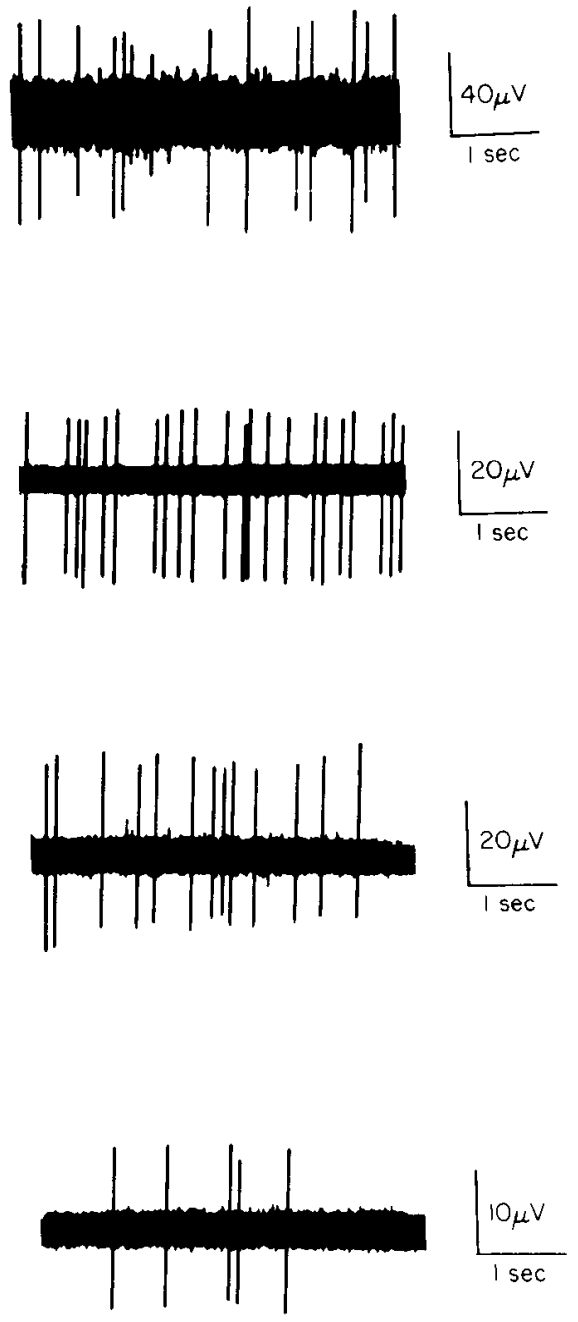

Figure 3. Response of $4 \mathrm{SCN}$ single units to photic stimulation. $A-C, \mathrm{Ex}-$ citatory responses to light. $D$, Inhibitory response. Changes in spike amplitude in $A$ (decreased) and $D$ (increased) may represent relative membrane depolarization and hyperpolarization, respectively.

\section{Post-mecamylamine response to photic stimulation}

Figure 4 shows the response of a single SCN cell to the various experimental manipulations. In Figure $4 C$, the photic response to light, post-mecamylamine, is absent. The overall absolute level of response to photic stimulation in both groups was greatly depressed following administration of mecamylamine $(t=1.6$, n.s. for SCN; $t=1.4$, n.s. for NONSCN). However, the signed $t$ response to light, after mecamylamine, is actually reversed in sign, as may be seen in the frequency histograms in Figure $6 \mathrm{~A}$. In other words, SCN cells, although less responsive to light in general after mecamylamine, showed, if anything, an inhibitory response to photic stimulation. The NONSCN cells, on the other hand, showed no such reversal in photic response following mecamylamine (Fig. 6B).

\section{Discriminant analysis}

A discriminant analysis was performed on the signed $t$ scores in order to determine whether the 4 dependent variables discussed above could jointly discriminate the 2 groups of anatomically defined cells. On the basis of this analysis, 2 groups of cells were distinguished, utilizing the dependent variables: (1) photic response, (2) nicotinic response, (3) mecamylamine response, and (4) post-mecamylamine photic response. The 2 groups of cells corresponded to histological hits within the SCN $(N=25)$ and misses beyond the border of the SCN $(N-23)$. Histological loci of the recording sites are shown in Figure 7, with symbols representing the nature of their response to photic or nicotinic stimulation. The ability of the discriminant function to distinguish these 2 cellular groups is graphically represented in Figure $8 A$. Action potentials recorded from the small group of probable chiasmatic fibers (see above) showed a response pattern (in terms of the dependent variables) that, in the discriminant analysis, classified them as members of the SCN group.

The accuracy of classification was approximately $90 \%$ for both groups. An analysis of posterior probabilities of group classification indicated that 2 SCN cells exhibited a constellation of responses more representative of the NONSCN group. Similarly, 2 NONSCN cells ( 1 in the anterior hypothalamus, 1 in the paraventricular region) showed response profiles similar to those of SCN neurons (i.e., visual response, nicotinic response, blockade by mecamylamine, attenuation or reversal of sign of visual response after mecamylamine).

The 4 dependent variables adequately discriminated between the 2 groups (canonical correlation $=0.72$, Hotelling $T^{2}=39.5$, $p<0.001)$. However, post-mecamylamine photic response exhibited by far the greatest discriminatory ability ( $F$ to enter $=$ $32.04, d f=1,50)$, followed by nicotinic response $(F$ to enter $=$ 
A.

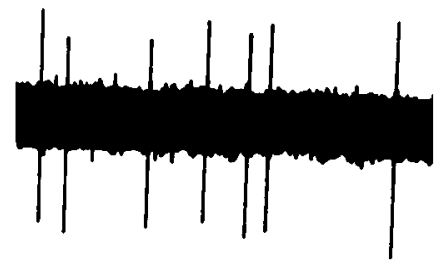

B.

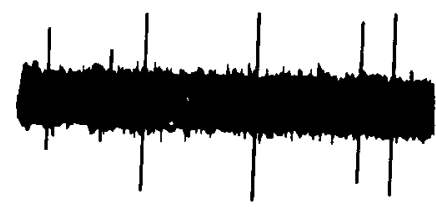

C. MECAMYLAMINE

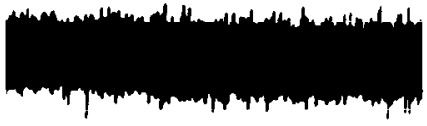

LIGHT

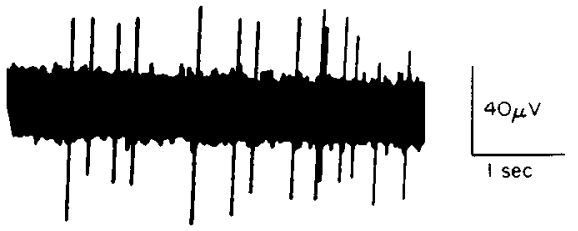

NICOTINE

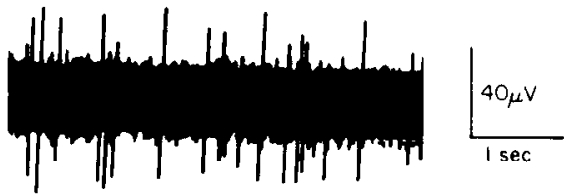

LIGHT AFTER MECAMYLAMINE

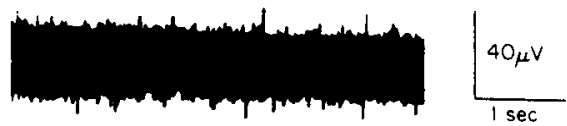

$6.41, d f=1,49)$, and pre-nicotine photic response $(F$ to enter $=$ $3.06, d f=1,48)$. The discriminatory ability of pre-nicotine photic response was probably underestimated by the stepwise analysis, due to the relatively high correlation with nicotinic response in the SCN group (see above). This interpetation was supported by an examination of the structure coefficients (prod-

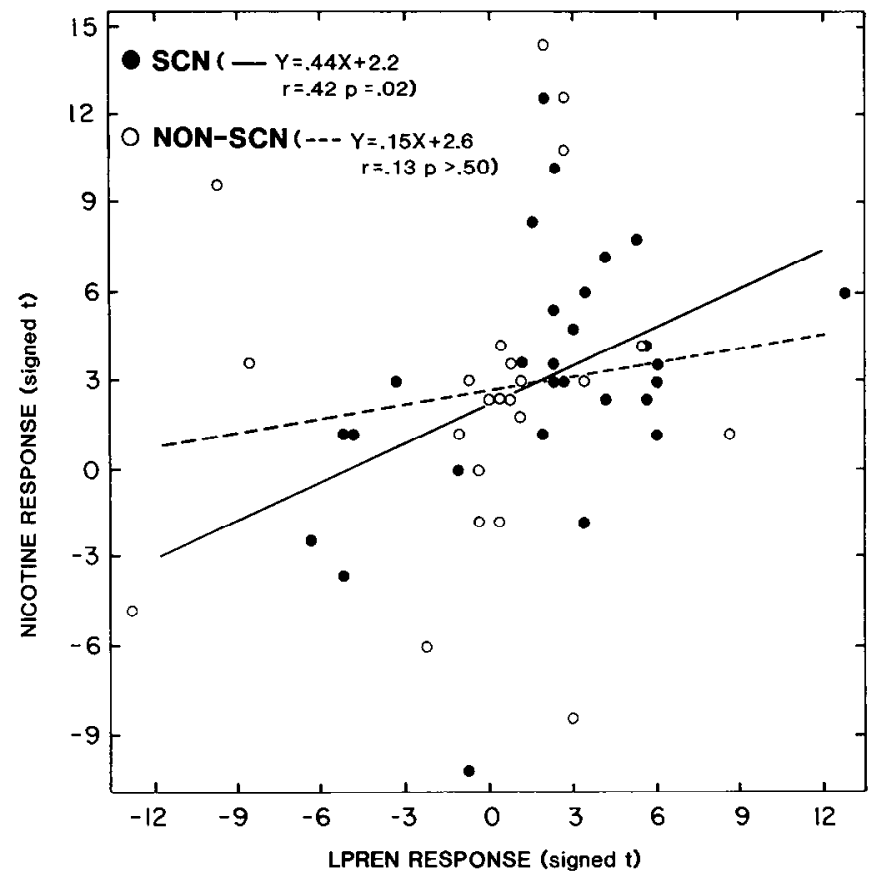

Figure 5. Scatter plots of the nicotinic response versus the pre-nicotine photic response ( $L P R E N)$ for $\mathrm{SCN}$ and NONSCN cells. The solid line is the regression line for placements in the $\mathrm{SCN}$, whereas the dashed line is the regression line for NONSCN placements. uct-moment correlations between the original 4 dependent variables and the constructed canonical variable or discriminant representing the weighted linear sum of the original variables best able to discriminate between the 2 groups). A plot of the canonical variate versus photic response before nicotine and after mecamylamine administration is shown in Figure 8, $B$ and $C$. It may be seen that both the pre-nicotine $(8 B)$ and postmecamylamine $(8 C)$ photic response variables contribute to the ability of the discriminant $(8 A)$ to separate the SCN and NONSCN groups. The canonical variable was positively correlated with the pre-nicotine photic response $(r=0.33)$ and highly negatively correlated with the post-mecamylamine visual response $(r=-0.87)$. This pattern suggested that a univariate repeated measures analysis of variance, with visual response as the replicate, would be of value (see below). Response to mecamylamine exhibited very little discriminative ability, which reflected its evaluation on the basis of change from pre-nicotine baseline, as opposed to its obvious antagonism of the immediately preceding nicotinic response.

\section{Analysis of variance}

Discriminant analysis (see above) suggested the value of a repeated measures analysis of variance for the SCN and NONSCN groups, with photic response (pre-nicotine and post-mecamylamine) as the replicate. Repeated measures analysis of variance indicated a strong recording locus $\times$ trial interaction $(F=11.84$, $p<0.001)$. Post hoc tests showed that mecamylamine treatment in the SCN group not only reduced the excitatory response to light, but actually drove it in an inhibitory direction $(p<0.001)$. That is, mecamylamine reduced excitatory responses and often converted them to inhibitory responses; inhibitory responses became more inhibitory (i.e., more negative). This trend was not obscrved in the NONSCN group. Histograms illustrating these phenomena for SCN and NONSCN cells are shown in 


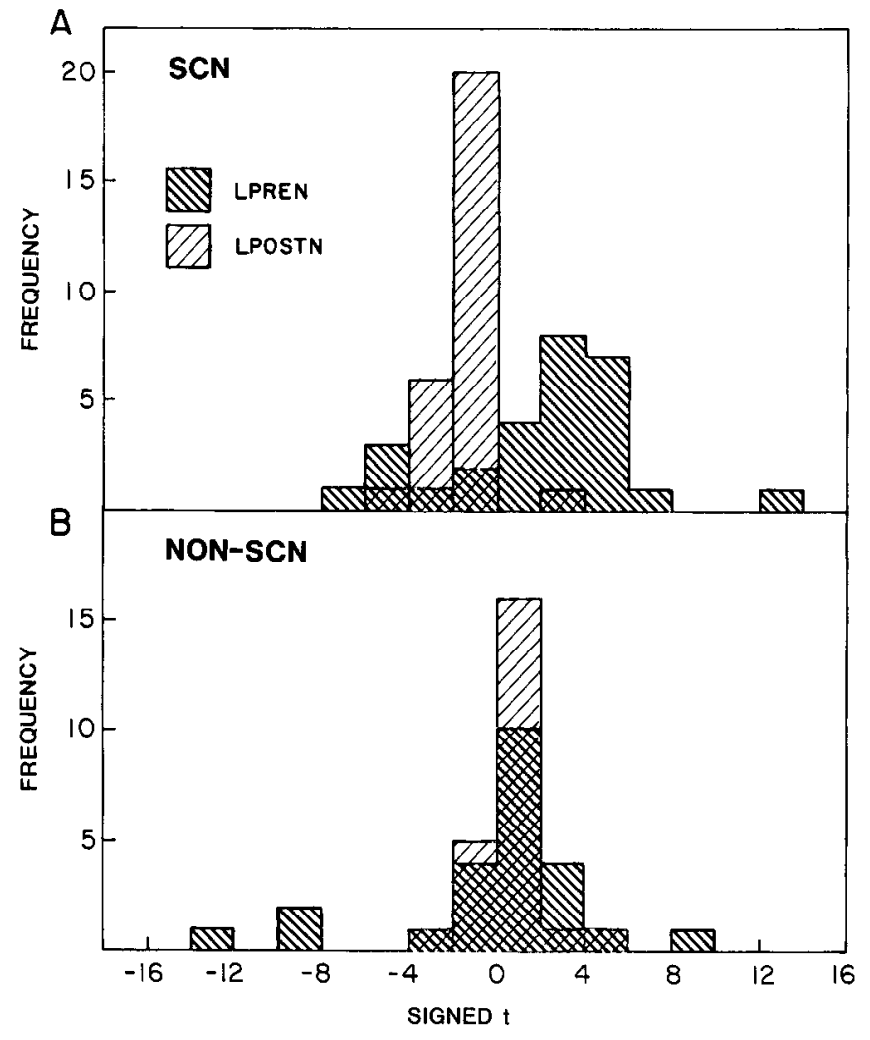

Figure 6. Univariate frequency histograms for $\mathrm{SCN}(A)$ and NONSCN $(B)$ groups on the dependent variables of pre-nicotine photic response $(L P R E N)$ and post-mecamylamine photic response (LPOSTN).
Figure 5. Figure 9 gives the means for the 2 groups on the replicate (photic response). It is apparent that the SCN cells are more strongly affected by antagonism of the nicotinic receptor.

\section{Discussion}

In summary, this study shows that both the photic and nicotinic responses of SCN neurons are typically excitatory. Nicotinic blockade eliminates the response to intravenous nicotine. Such blockade either eliminates the visual response of these neurons or may even drive it in an inhibitory direction. Reduction of absolute levels of photic responsiveness was also observed in certain of the small number of NONSCN cells that were photically responsive initially, but the trend to augmented inhibition after mecamylamine appeared to be characteristic of the SCN.

\section{Photic response}

In agreement with other authors, this study has demonstrated that SCN cells are often highly responsive to light. In addition, cells beyond the borders of the SCN as defined by Nissl histochemistry are occasionally responsive to light, in agreement with observations showing that the retinohypothalamic tract (RHT) may terminate in regions beyond the Nissl-defined borders of the SCN (Fuller et al., 1984). In agreement with other authors, we found that the visual response in SCN and peri-SCN regions is typically excitatory.

\section{Nicotinic response}

Nicotinic response is quite widespread within and beyond the borders of the SCN. This finding is in excellent agreement with recent histochemical results showing that $\mathrm{AChE}$ is relatively
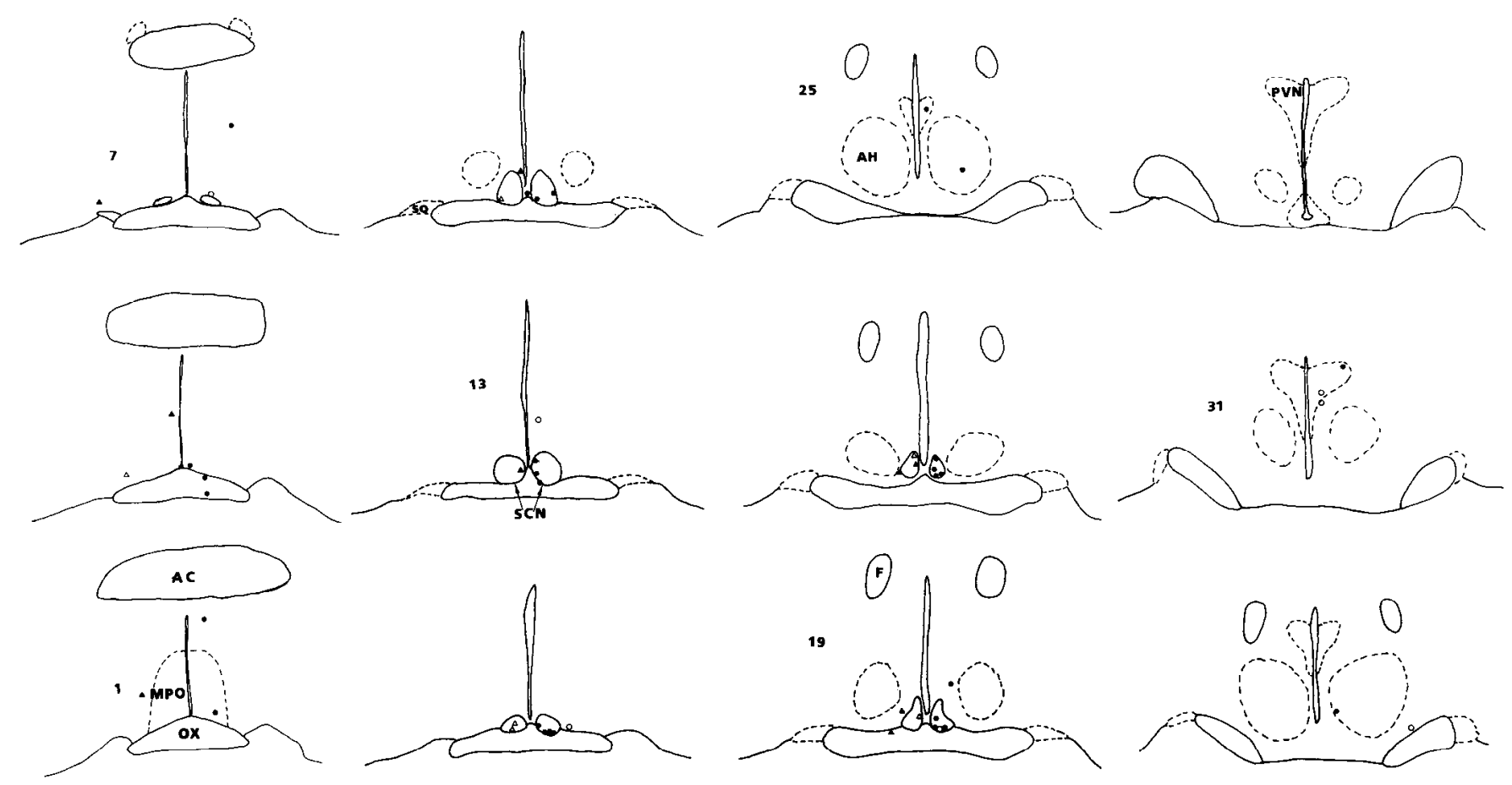

Figure 7. Recording loci in the hypothalamus. Every third coronal section $(40 \mu \mathrm{m})$, ordered anterior to posterior, is shown. Numbers at left are section numbers; symbols at right of each section indicate cells jointly responsive to light and nicotine (O) or cells unresponsive to light and nicotine $(O)$. Symbols at left indicate exclusively nicotine-responsive $(\triangle)$ or light-responsive (A) cells. $A C$, anterior commissure; $M P O$, medial preoptic nucleus; $O X$, optic chiasm; $S C N$, suprachiasmatic nucleus; $S O$, supraoptic nucleus; $A H$, anterior hypothalamic nucleus; $F$, fornix; $P V N$, paraventricular hypothalamic nucleus. 
A

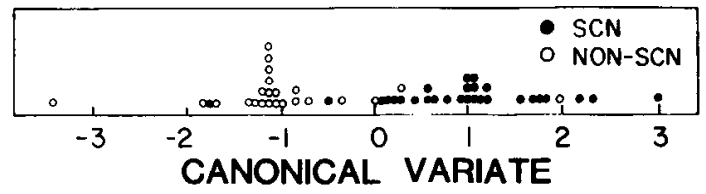

B
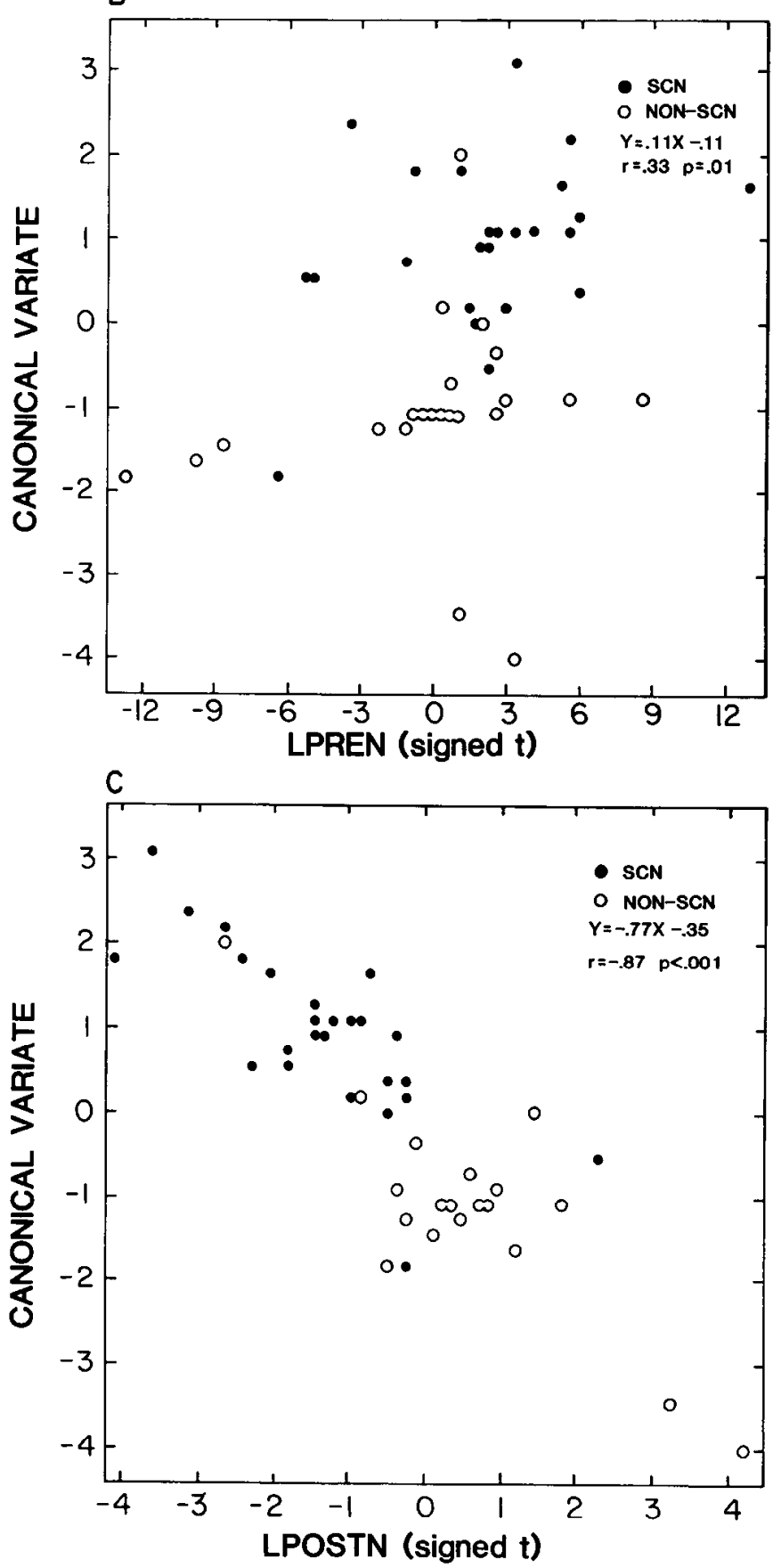

Figure 8. A, Histogram of the discriminant function representing the weighted linear sum of the original 4 dependent variables best able to discriminate the 2 cellular groups (SCN, NONSCN). B, Plot of the canonical variate versus the visual response before nicotine (LPREN). $C$, Plot of the canonical variate versus the visual response after mecamylamine (LPOSTN).
LOCUS $X$ TRIALS

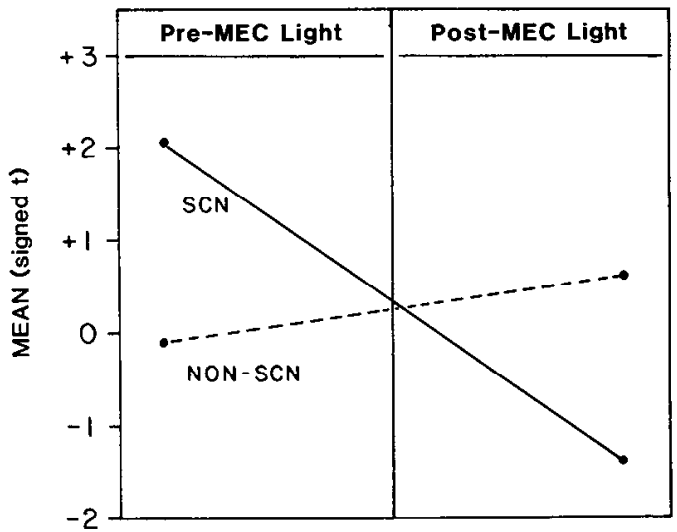

Figure 9. Locus $\times$ Trials interaction. The mean response of SCN cells to photic stimulation changed sign after nicotinic blockade. The mean response of non-SCN cells to photic stimulation was small initially and relatively unaffected by nicotinic blockade.

widely dispersed in the SCN and peri-SCN regions (Lauber et al., 1984), and with autoradiographic (Segal et al., 1978) and immunohistochemical data (Mason, 1985; Ichikawa and Hirata, 1986) on the distribution of the putative nicotinic receptor in this brain region.

\section{Photic-nicotinic correlation}

Nicotinic response is quite highly correlated with visual response in the SCN. Again, our histochemical results show a considerable degree of overlap between RHT terminals and AChE-labeled neurons in this region (Lauber et al., 1984). It would appear that visually responsive neurons in the SCN will typically be nicotine responsive, although the converse may not be true, owing to the observation that $\mathrm{AChE}$-labeled neurons appear to be present in regions lacking an RHT input. Indeed, Nishino and Koizumi (1977) have reported that over $90 \%$ of SCN cells not showing a response to optic tract stimulation still exhibit an excitatory response to iontophoretically administered ACh.

The initial inhibitory responses occasionally observed to light or nicotine may have reflected stimulation-induced depolarization inactivation. Depolarization inactivation is associated with both a reduction in extracellular spike amplitude and a decrease in firing rate, both of which were occasionally observed in this study. Thus, a uni-process model may explain both the excitatory and less frequent "inhibitory" responses to photic or nicotinic activation. When an SCN neuron is firing somewhere around the middle of its dynamic range, further depolarization should increase firing rate. If the cell is firing near the high end of its dynamic range, further depolarization may result in depolarization inactivation, amplitude reduction, and subsequent decrease in firing rate. On the other hand, it is possible that the reversal of sign in the response to photic stimulation seen in some cells after nicotinic receptor blockade may represent a true hyperpolarizing response to light, "revealed" by nicotinic blockade. Only intracellular recording can unambiguously determine the effects of visual stimulation and nicotinic activation on the membrane potential of the SCN neuron. Indeed, recent results (Wheal and Thomson, 1984) indicate that, in the vast majority 
of SCN neurons recorded intracellularly, the response to optic tract stimulation is depolarizing. Thus, available intracellular data appear to support the uni-process model discussed above. Single-oscillator models of circadian function that posit 2 populations of SCN neurons-one excited and the other inhibited by photic stimulation (e.g., Carpenter and Grossberg, 1985)may therefore require revision.

\section{Mecamylamine response}

Mecamylamine is an effective nicotinic antagonist in the SCN region. It appeared to have a normalizing effect on SCN firing rates altered by nicotine administration, in addition to its ability to block the effects of subsequent doses of nicotine.

\section{Post-mecamylamine response}

Certain of the results of this investigation may be explained either by post- or presynaptic nicotinic receptor mechanisms. An exclusive postsynaptic interpretation is made less likely by the electrophysiological responses seen after mecamylamine. Blockade of the nicotinic receptor either abolished, or even reversed the sign of, the unit response to photic stimulation. Since this effect was observed in numerous cells in which baseline firing rate was not altered by mecamylamine, it is unlikely that this effect can be entirely explained via the influence of a nicotinic postsynaptic receptor on the SCN neuron (although we cannot exclude a modulatory role of such a receptor in at least some cases). Furthermore, discriminant analysis classified chiasmatic fibers with the SCN group, suggesting that presynaptic clements can show the entire constcllation of effects characteristic of the postsynaptic SCN neuron. Thus, it is possible that the RHT is "gated out" by nicotinic blockade via a presynaptic mechanism. However, since ocular enucleation does not influence the distribution of AChE labeling in the SCN (Lauber et al., 1984), it is doubtful that such a presynaptic nicotinic receptor resides on RHT terminals. Furthermore, axoaxonal synapses in the SCN are rare or nonexistent (Guldner, 1978). The strong implication is that nicotinic stimulation and blockade may modulate the transmission of visual information to the SCN via a direct effect at the retinal level. Nicotinic receptors have been both pharmacologically (Vogel et al., 1977; Pourcho, 1979) and physiologically (Masland and Ames, 1976; Ariel and Daw, 1982a) characterized on retinal ganglion cells in the rabbit. Such cells are exquisitely responsive to nicotine, and the nicotinic responses are readily blocked by antagonists such as mecamylamine (Ariel and Daw, 1982b). Cholinergic responses are not, however, readily antagonized by the muscarinic blocker, scopolamine (Masland and Ames, 1976). It appears that cells with On-center receptive fields typically exhibit enhanced responses to light under cholinergic stimulation, whereas Off-center cells are relatively refractory (Masland and Ames, 1976; Masland, 1980). Tonically responsive, large-field, and sluggish ganglion cells-which may represent a major afferent input to the cat SCN (Groos and Mason, 1980; Fuller et al., 1983)-are particularly sensitive to this form of neuromodulation (Ariel and Daw, 1982a). Perhaps specific blockade of retinal ganglion cell on responses by mecamylamine, with relative sparing of Off responses, may explain the shift to inhibitory response, seen under photic stimulation, of the SCN cells observed in this study. Whether this inhibitory response to light, under nicotinic blockade, is truly hyperpolarizing can only be determined by future intracellular studies.

In spite of the explanatory power of the retinal hypothesis discussed above, we cannot exclude a contributory role for the cholinergic receptor on SCN cell bodies, in terms of the electrophysiological effects discussed here, or other observations indicating a role for such a receptor in certain visually mediated phenomena (e.g., Zatz and Brownstein, 1981). It appears unlikely, however, that a nicotinic receptor remote from the retinohypothalamic pathway can explain the constellation of results seen here. Such a receptor would be required to mediate the blockade or alteration of visual response in both SCN neurons and presynaptic elements (RHT axons). It is difficult to see how such a remote mechanism could explain the alterations in RHT axonal impulse flow observed in this study following nicotinic blockade, since there are no characterized retinopetal projections in the mammalian CNS and there are few, if any, axoaxonal synapses in the SCN (Guldner, 1978). Furthermore, when axoaxonal synapses do occur, they control transmitter release rather than axonal impulse flow. Finally, in the case of the postsynaptic SCN neuron, we know of no mechanism by which the nicotinic blockade of a receptor on some remote neuron, projecting to the SCN neuron, could alter the photic response of the SCN neuron, in the absence of a change in baseline firing rate. In any event, it is probable that the common effect of either cholinergic or visual stimuli is a net activation of the SCN, resulting in phase shifts of overt circadian rhythms not unlike those produced by direct electrical stimulation of the SCN.

\section{Conclusion}

The effects of cholinergic agonists and antagonists on behavioral and neurochemical circadian rhythms (Zatz and Brownstein, 1981; Zatz and Herkenham, 1981; Earnest and Turek, 1985) might be mediated by pre- or postsynaptic mechanisms in the RHT. The results reported here suggest that a cholinergic receptor, either at the level of the RHT terminal in the SCN, or in the retina, may be of primary importance for the phase-shift effects of cholinergic agonists on circadian rhythms. The paucity of axoaxonal terminals in the SCN (but see Mason, 1985) suggest that the prime site of action of cholinergic agents may be on cholinergic receptors on retinal ganglion cells projecting to the SCN. In any event, the results reported here indicate that such a receptor, regardless of its location, is probably nicotinic in nature.

A final point is that the visual and/or nicotinic response of SCN neurons may represent a rather early step in visual information processing in the SCN, quite possibly considerably removed from those intrinsic timing mechanisms that ultimately generate circadian rhythmicity. At least 2 instances of dissociation between photic response and circadian processes have been noted. Spontaneous unit activity in the SCN varies lawfully through the light-dark cycle (Green and Gillette, 1982; Shibata et al., 1982), but the evoked response of single neurons to visual stimulation does not vary in a circadian fashion (Inouye, 1984). In addition, intra-SCN administration of TTX blocks behavioral response to a visual Zeitgeber but does not alter the period or phase of the endogenous pacemaker (Schwartz et al., 1985). Future studies are necessary to identify the mechanisms through which visual information modifies the intrinsic SCN pacemaker. 


\section{References}

Ariel, M., and N. W. Daw (1982a) Effects of cholinergic drugs on receptive field properties of rabbit retinal ganglion cells. J. Physiol. (Lond.) 324: 135-160.

Ariel, M., and N. W. Daw (1982b) Pharmacological analysis of directionally sensitive rabbit retinal ganglion cells. J. Physiol. (Lond.) 324: $161-185$

Block, G. A., and R. B. Billiar (1981) Properties and regional distribution of nicotinic cholinergic receptors in the rat hypothalamus. Brain Res. 212: 152-158.

Carpenter, G. A., and S. Grossberg (1985) A neural theory of circadian rhythms: Split rhythms, after-effects, and motivational interactions. J. Theor. Biol. 113: 163-223.

Earnest, D. J., and F. W. Turek (1985) Neurochemical basis for the photic control of circadian rhythms and seasonal reproductive cycles: Role for acetylcholine. Proc. Natl. Acad. Sci. USA 82: 4277-4281.

Fuller, C. A., D. M. Murakami, and J. D. Miller (1983) Retinal ganglion cell projection to the suprachiasmatic nucleus in the cat and rat. Soc. Neurosci. Abstr. 9: 1067.

Fuller, C. A., D. M. Murakami, and J. D. Miller (1984) Retinal ganglion cell projections and cytochrome oxidase activity within the hypothalamus of the rat and cat. Soc. Neurosci. Abstr. 10: 503.

Green, D. J., and R. Gillette (1982) Circadian rhythm of firing rate recorded from single cells in the rat suprachiasmatic brain slice. Brain Res. 245: 198-200.

Groos, G., and R. Mason (1978) Maintained discharge of rat suprachiasmatic neurons at different adaptation levels. Neurosci. I ett. 8 : $59-64$.

Groos, G., and R. Mason (1980) The visual properties of rat and cat suprachiasmatic ncurons. J. Comp. Physiol. 135: 349-356.

Guldner, F. H. (1978) Synapses of optic nerve afferents in the rat suprachiasmatic nucleus. Cell Tissue Res. 194: 17-35.

Ichikawa, T., and Y. Hirata (1986) Organization of choline acetyltransferase containing structures in the forebrain of the rat. J. Neurosci. 6: 281-292.

Inouye, S. T. (1984) Light responsiveness of the suprachiasmatic nucleus within the island with the retino-hypothalamic tract spared. Brain Res. 294: 263-268.

Inouye, S. T., and H. Kawamura (1982) Characteristics of a circadian pacemaker in the suprachiasmatic nucleus. J. Comp. Physiol. 146: 153-160.

Kreisel, B., N. Conforti, M. Gutnick, and S. Feldman (1979) Suprachiasmatic nucleus responsiveness to photic and basal hypothalamic stimulation. Brain Res. Bull. 3: 707-714.

Lauber, A. H., J. D. Miller, D. M. Murakami, and C. A. Fuller (1984) Cholinoceptive neurons of the SCN. Soc. Neurosci. Abstr. 10: 501.

Masland, R. H. (1980) Acetylcholine in the retina. In Neurochemistry of the Retina, N. G. Bazan and R. N. Lolley, eds., pp. 501-518, Pergamon, New York.

Masland, R. H., and A. Ames (1976) Responses to acetylcholine of ganglion cells in an isolated mammalian retina. J. Neurophysiol. 39: 1220-1235.

Mason, W. T. (1985) Staining of the magnocellular nuclei of the rat hypothalamus by a monoclonal antibody directed against the subunit of the nicotinic cholinergic receptor. Neurosci. Lett. 59: 89-95.

Moore, R. Y., and V. B. Eichler (1976) Central neural mechanisms in diurnal rhythm regulation and neuroendocrine responses to light. Psychoneuroendocrinology 1: 265-279.

Moore-Ede, M. C., F. M. Sulzman, and C. A. Fuller (1982) The Clocks That Time Us, Harvard University Press, Cambridge, MA.

Murakami, N., K. Takahashi, and K. Kawashima (1984) Effect of light on the acetylcholine concentrations of the suprachiasmatic nucleus in the rat. Brain Res. 311: 358-360.

Nishino, H., and K. Koizumi (1977) Responses of neurons in the suprachiasmatic nuclei of the hypothalamus to putative transmitters. Brain Res. 120: 167-172.

Nishino, H., K. Koizumi, and C. McChandler Brooks (1976) The role of the suprachiasmatic nucleus of the hypothalamus in the production of circadian rhythm. Brain Res. 112: 45-59.

Paxinos, G., and C. Watson (1982) The Rat Brain in Stereotaxic Coordinates, Academic, New York.

Pourcho, R. G. (1979) Localization of cholinergic synapses in mammalian retina with peroxidase conjugated $\alpha$-bungarotoxin. Vision Res. 19: $287-292$.

Rusak, B. (1977) Involvement of the primary optic tracts in mediation of light effects on hamster circadian rhythms. J. Comp. Physiol. 118: $165-172$.

Rusak, B., and G. Groos (1982) Suprachiasmatic stimulation phase shifts rodent circadian rhythms. Science 215: 1407-1409.

Sawaki, Y. (1979) Suprachiasmatic nucleus neurones: Excitation and inhibition mediated by the direct retino-hypothalamic projection in female rats. Exp. Brain Res. 37: 127-138.

Schwartz, W. J., R. A. Gross, and M. T. Morton (1985) The suprachiasmatic nuclei contain a tetrodotoxin-resistant circadian pacemaker. Soc. Neurosci. Abstr. 11: 818.

Segal, H., Y. Dudai, and A. Amsterdam (1978) Distribution of an $\alpha$-bungarotoxin binding cholinergic nicotinic receptor in rat brain. Brain Res. 148: 105-119.

Shibata, S., Y. Oomura, H. Kita, and K. Hattori (1982) Circadian rhythmic changes of neuronal activity in the suprachiasmatic nucleus of the rat hypothalamic slice. Brain Res. 247: 154-158.

Stephan, F. K., and I. Zucker (1972) Circadian rhythms in drinking behavior and locomotor activity of rats are eliminated by hypothalamic lesions. Proc. Natl. Acad. Sci. USA 69: 1583-1586.

Vogel, Z., G. J. Maloney, A. Ling, and M. P. Daniels (1977) Identification of synaptic acetylcholine receptor sites in retina with peroxidase-labeled $\alpha$-bungarotoxin. Proc. Natl. Acad. Sci. USA 74: 3268.

Wheal, H. V., and A. M. Thomson (1984) The electrical properties of neurones of the rat suprachiasmatic nucleus recorded intracellularly in vitro. Neuroscience 13: 97-104.

Winer, B. J. (1962) Statistical Principles in Experimental Design, McGraw-Hill, New York.

Zatz, M., and M. J. Brownstein (1981) Injection of $\alpha$-bungarotoxin near the suprachiasmatic nucleus blocks the effects of light on nocturnal pineal enzyme activity. Brain Res. 213: 438-442.

Zatz, M., and M. A. Herkenham (1981) Intraventricular carbachol mimics the phase-shifting effect of light on the circadian rhythm of wheel-running activity. Brain Res. 212: 234-238. 